\title{
Negative Regulation of Immunologic Factor Signaling
}

National Cancer Institute

\section{Source}

National Cancer Institute. Negative Regulation of Immunologic Factor Signaling. NCI

Thesaurus. Code C40593.

Any physiologic process which occurs in response to ligation of an immunologic factor receptor with its cognate ligand, and results in a reduced number of active signaling molecules within the target cell. This process is involved in regulating the extent of the response to receptor binding, as well as regulating active populations of immune cells. 